\title{
Prevalence of Nonadherence With Maintenance Mesalamine in Quiescent Ulcerative Colitis
}

\author{
Sunanda V. Kane, M.D., M.S.P.H., Russell D. Cohen, M.D., James E. Aikens, Ph.D., and
}

Stephen B. Hanauer, M.D.

Department of Medicine, University of Chicago, Chicago, Illinois; and Department of Family Medicine, University of Michigan, Ann Arbor, Michigan

OBJECTIVE: There are scant data regarding outpatient adherence in quiescent ulcerative colitis aside from patients enrolled in controlled clinical trials. We conducted a prevalence study to determine the medication adherence rate of maintenance therapy and to identify possible risk factors for nonadherence.

METHODS: Outpatients with clinically quiescent ulcerative colitis for $>6$ months on maintenance mesalamine (Asacol, Procter and Gamble, Cincinnati, $\mathrm{OH}$ ) were eligible. Patients were interviewed regarding disease history, and demographics were obtained from medical records. Refill information for at least 6 months was obtained from computerized pharmacy records. Adherence was defined as at least $80 \%$ consumption of supply dispensed. Using nonadherence as the outcome of interest, stratified analysis and regression modeling were used to identify significant associations.

RESULTS: Data were complete for the 94 patients recruited. The overall adherence rate was found to be $40 \%$. The median amount of medication dispensed per patient was $71 \%(8-130 \%)$ of the prescribed regimen. Nonadherent patients were more likely to be male $(67 \%$ vs $52 \%, p<$ $0.05)$, single $(68 \%$ vs $53 \%, p=0.04)$, and to have disease limited to the left side of the colon versus pancolitis (83\% vs $51 \%, p<0.01)$. Sixty-eight percent of patients who took more than four prescription medications were found to be nonadherent versus only $40 \%$ of those patients taking fewer medications $(p=0.05)$. Age, occupation, a family history of inflammatory bowel disease, length of remission, qualityof-life score, or method of recruitment (telephone interview $v s$ clinical visit) were not associated with nonadherence. Logistic regression identified that a history of more than four prescriptions (odds ratio [OR] 2.5 [1.4-5.7]) and male gender (OR 2.06 [1.17-4.88]) increased the risk of nonadherence. Two statistically significant variables, which were protective against nonadherence, were endoscopy within the past 24 months (OR 0.96 [0.93-0.99]) and being married (OR 0.46 [0.39-0.57]).

CONCLUSION: Nonadherence is associated with multiple concomitant medications, male gender, and single status. These patient characteristics may be helpful in targeting those patients at higher risk for nonadherence. (Am J Gas- troenterol 2001;96:2929-2933. (C) 2001 by Am. Coll. of Gastroenterology)

\section{INTRODUCTION}

Ulcerative colitis (UC) is an idiopathic, chronic inflammatory disease of the large intestine characterized by episodes of relapse and remission. Relapses are often not predictable, although factors such as smoking cessation $(1,2)$, chronic nonsteroidal anti-inflammatory use $(3,4)$, and psychological stress (5) are thought to cause symptom exacerbation in some individuals.

Because of its chronic nature, therapy often must continue on an indefinite basis. Multiple studies have demonstrated the efficacy of aminosalicylates to induce and maintain remission in UC (6-10). These well-designed, multicenter trials have used pill count and patient inquiry to assess adherence, with rates ranging from $70 \%$ to $>95 \%$. This wide variance across studies can be explained by the study's definition of adherence, the degree to which the investigators were proactive in adherence measures, and patient population. Based upon community-based, follow-up studies in other chronic illnesses, the percentage of long-term adherence tends to be much lower, about $40-50 \%$ (11).

Despite the advances in treatment modalities, many patients experience relapses. Many patients openly admit they do not take their medications as prescribed. Medication taking probably makes patients more uncomfortably aware of their chronic illness status. They have a fear of long-term side effects from medications, and they question the need for medication in the setting of quiescent disease. However, the impact of medication adherence on specific inflammatory bowel disease (IBD) outcomes has not been fully explored. The purpose of this point-prevalence study was first to determine the prevalence of adherence among patients taking mesalamine to prevent relapse in quiescent $\mathrm{UC}$, and then determine those risk factors associated with nonadherent behavior.

\section{MATERIALS AND METHODS}

\section{Patients}

Consecutive patients were recruited from the Gastroenterology Clinic at the University of Chicago from March to 
October, 1998. Potential patients were identified from three sources: patient clinic visits, patient phone requests for refills of Asacol, or pharmacy calls for Asacol. Those patients with a well-established diagnosis of UC confirmed by endoscopy and histology were eligible. Entry criteria included patients in clinical remission at least 6 months from the time of potential recruitment. Remission was defined by criteria modified from Truelove and Witts: one to three formed bowel movements per day without bleeding, urgency, or cramping (12). Patients also had to have documentation of Asacol use without concurrent oral or topical steroids, or other immunomodulators such as azathioprine or 6-mercaptopurine to maintain remission. Exclusion criteria included any evidence of clinically active disease in the previous 6 months, no documented visit to a physician in our clinic in the previous 18 months, or uncertainty of the diagnosis (Crohn's disease or indeterminate colitis).

\section{Protocol}

Retrospective data for each patient was collected during a single interview. For patients recruited from pharmacy calls to the office, refill data were obtained, and the medical records verified the patient's diagnosis. The patient was called to confirm the refill and collect data on current clinical status. For those patients who called the office to request a refill, the pharmacy data were collected at the time of the refill, the patient was contacted to confirm the refill and obtain clinical information. For patients recruited from the clinic, clinical data were collected during the patient interview, the medical record reviewed for any missing information, and then the pharmacy was contacted for refill data.

Demographic variables collected included age, gender, marital status, occupation, and type of insurance. Disease information included extent (proctitis, left-sided, pancolitis) and duration of disease, length of present remission, mesalamine dose and regimen, family history of IBD, and concomitant medications. The length of time since last steroid use, last hospitalization, and last colonoscopy were also collected. A formal global assessment score including patient well-being was not computed; however, quality of life was measured using the Short Inflammatory Bowel Disease Questionnaire (13). Data to compute this score were obtained during the patient interview. To avoid acquisition bias, all information was collected by one investigator (S.V.K.), and information regarding clinical status was obtained before and separate from MED-TOTAL calculations (see below).

\section{Statistical Analysis}

Adherence was calculated using Steiner's "MED-TOTAL" formula, which has been validated in plasma drug levels (phenytoin) and medical outcomes (diastolic blood pressure) (14). Consumption of $>80 \%$ of prescribed medications over a 6-month period was used as the definition of "adherence." We chose this percentage based on the principle that loss of $>20 \%$ of a patient population in a clinical
Table 1. Patient Characteristics

\begin{tabular}{lc}
\hline \multicolumn{1}{c}{ Variable } & $\begin{array}{c}\text { Median } \\
\text { (Range) }\end{array}$ \\
\hline Age (yr) & $42.5(18-79)$ \\
Gender $(\%$ male) & 51 \\
Yr disease & $8(1-66)$ \\
Length remission (mo) & $24(6-360)$ \\
SIBDQ score & $61(45-70)$ \\
Dose (g/day) & $3.88(1.2-4.8)$ \\
Married $(\%)$ & 53 \\
Adherence $(\%)$ & 40 \\
\hline
\end{tabular}

SIBDQ $=$ Short Inflammatory Bowel Disease Questionnaire.

trial makes the results suspect and potentially invalid (15). MED-TOTAL was calculated as:

$$
\frac{\text { Sum of days' supply dispensed }}{\text { Sum of days in all refill intervals }} \times 100
$$

The outcome variable of interest was adherence. $\chi^{2}$ testing, or McNemar's test where appropriate, was performed to compare frequencies of demographic variables and disease characteristics between adherent versus nonadherent groups. Multivariate logistic regression modeling was performed to identify independent variables that correlated with nonadherent behavior. Measurement of risk associated with nonadherence using odds ratios (OR) was calculated along with $95 \%$ CI. A $p$ value of 0.05 was considered statistically significant. All tests were two-tailed. The protocol was approved by the University of Chicago Institutional Review Board before the collection of any data.

\section{RESULTS}

Ninety-four consecutive patients were enrolled in the cohort; their characteristics are shown in Table 1. The median age was $42.5,51 \%$ were men. Nearly half of the patients (47\%) had pancolitis with a median duration of disease of 8 yr. The median mesalamine dose was $3.9 \mathrm{~g} /$ day. Sixty-four patients were recruited from the clinic, 20 from direct pharmacy calls, and 10 via patient telephone refill requests.

We found that less than half of the patients $(40 \%)$ were adherent to their prescribed dose of mesalamine. The median amount of medication consumed was $71 \%$ (range $8-100)$ of the total prescribed dose.

There was a variety of dosing regimens used, but because the majority of patients were taking b.i.d. mesalamine $(67 \%$ of the patients), dosing regimen was stratified as a dichotomous variable (b.i.d. vs other). Stratification by prescribed dose or dosing regimen failed to reveal any significant differences in adherence rates (Figs. 1 and 2). When stratified by extent of disease, the trend toward nonadherence in those with less extensive disease was significant at $p=0.04$.

Univariate analysis identified four factors, which were significantly associated with nonadherence: male gender, single status, a diagnosis of left-sided disease, and a history of more than four concomitant medications. Logistic regres- 


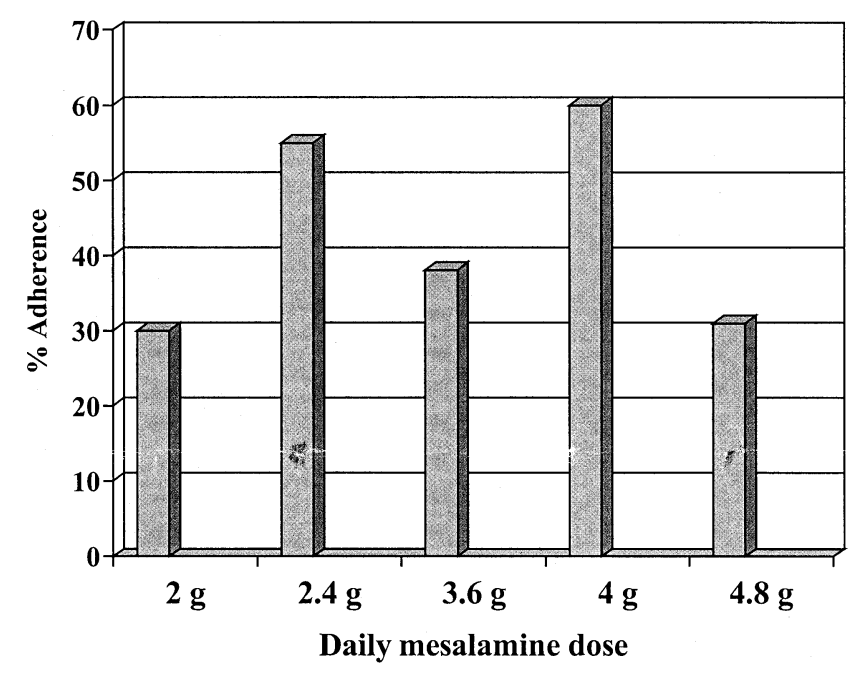

Figure 1. Percent adherence by prescribed dose. $p=0.53$.

sion found that men were twice as likely to be nonadherent than women (OR 2.1, 95\% CI $=1.2-4.8)$, and that a history of more than four concomitant medications was associated with a two and a half times increased chance of nonadherence with mesalamine (OR 2.5, 95\% CI $=1.4-5.7)$. Conversely, being married, having had a recent colonoscopy, and a greater extent of disease were all positively associated with adherence (see Table 2).

\section{DISCUSSION}

We found when using objective pharmacy data, rather than patient-derived information, the prevalence of medication nonadherence was $60 \%$ in patients with quiescent UC. The average amount of medication consumed was $70 \%$ of that prescribed. These numbers are comparable with those seen in asymptomatic phases of other chronic illnesses (11).

There are several published studies in the English literature addressing medication adherence in UC. van Hees and van Tongeren (16) measured urine levels of acetylated sul-

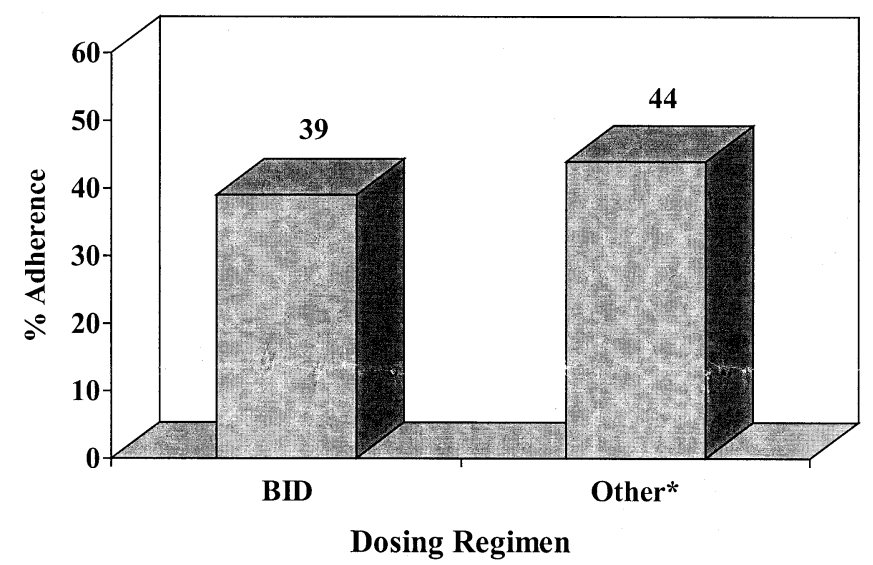

Figure 2. Percent adherence by regimen. $p=0.39$. $* 27 \%$ three times a day, $6 \%$ four times a day dosing. fasalazine as a marker for adherence in 51 patients 1-6 months after hospital discharge and in 171 outpatients over several years. The authors concluded that nonadherence, as defined by undetectable urine levels, in the months after hospital discharge approximated 41.2\%. In 175 patients followed on maintenance doses of sulfasalazine, $12 \%$ had undetectable urine levels at 6-month follow-up.

In another study, Riley et al. included adherence as a potential factor leading to disease relapse (17). Medication adherence was determined by pill count and direct patient inquiry. After $48 \mathrm{wk}$, there was no difference in adherence rates between the group who relapsed versus those who remained in remission. However, the total adherence for both groups was $>95 \%$ throughout the study, making the interpretation of these findings difficult.

Because patients are implying consent to contact their pharmacy for refills, we believed that this was the most accurate reflection of medication consumption possible outside of a structured clinical trial. We assumed that the number of pills dispensed to a patient over a certain time interval was an accurate estimate of pill consumption. It is believed that this assumption is valid because it is unlikely that patients will pick up medications they have no intention of taking. We collected data on at least 6 months' worth of medication refills. This would include at least two-time intervals for those patients who are dispensed medications for a 90-day period, and would minimize any bias associated with a patient either "stockpiling" or self-medicating unbeknownst to the prescribing physician. It is also not the habit of prescribing physicians at our institution to prescribe more medication than deemed necessary to maintain remission to ensure medication adherence.

There is no guarantee that our patients did not get medications from several physicians. However, we tried to minimize this possibility by asking the pharmacist about all prescriptions for mesalamine filled over the previous 6 months, regardless of physician name. In addition, all of the pharmacies on record for each patient were contacted (i.e., the one close to work, the one close to home, etc.). In the Chicago land area, the larger chains of commercial drug stores are linked via a computer system, and so refills obtained from multiple locations can be tracked.

Direct inquiry is a standard method for measuring adherence, but for a variety of reasons patients are untruthful when a physician asks patients if they are taking their medication $(18,19)$. These include the fear of displeasing their physician or shame or embarrassment regarding inability to pay for medication. Pill counts for maintenance therapy are cumbersome and do not necessarily reflect the true medication consumption rates in outpatients.

One of the strengths of this study was the use of the Short Inflammatory Bowel Disease Questionnaire to standardize the severity of each patient's perceived symptoms. Also, there was no "nonresponder" bias as all eligible consecutive patients were included. In addition, we tried to minimize any 
Table 2. OR for Risk Factors for Nonadherence

\begin{tabular}{llll}
\hline & OR & $95 \%$ CI & \\
\hline Male gender & 2.1 & $1.2-4.8$ & 0.02 \\
More than four concomitant medications & 2.5 & $1.4-5.7$ & 0.02 \\
Married status & 0.46 & $0.39-0.57$ & 0.01 \\
Greater extent of disease & 0.55 & $0.22-1.33$ & 0.07 \\
Recent colonoscopy & 0.96 & $0.93-0.99$ & 0.03 \\
Age & 1.07 & $0.91-2.77$ & 0.28 \\
Yr disease & 1.9 & $0.56-7.4$ & 0.50 \\
SIBDQ score & 0.99 & $0.94-1.05$ & 0.96 \\
Dose & 0.88 & $0.61-1.22$ & 0.53 \\
Regimen & 1.32 & $0.52-3.33$ & 0.55 \\
Length of remission & 1.41 & $0.78-4.56$ & 0.51 \\
Recruitment method & 1.01 & $0.60-2.10$ & 0.44 \\
\hline
\end{tabular}

Abbreviation as in Table 1.

selection bias by using several methods for patient recruitment.

It is difficult to get patients to take medication when they feel well, because the rationale for continued consumption is unclear. The long-term goals of improving adherence are to reduce frequency of relapse, lower the incidence of longterm complications (i.e., colon cancer), and lower overall health costs.

The consequences of nonadherence are self-evident in other illnesses such as coronary heart disease and diabetes, but not so for IBD. There are now several studies that suggest that documented medication consumption is protective for colon cancer, which is an important concern for the long-term natural history of UC. Moody et al. studied 168 patients with UC diagnosed between 1972 and 1981 and correlated nonadherence with sulfasalazine with risk of colorectal cancer (20). Their crude colectomy rate was $23 \%$ in $10 \mathrm{yr}$ with a $3 \%$ rate in those patients on maintenance sulfasalazine, and $31 \%$ in patients either noncompliant or off all medications. The study was a chart review, so nonadherence was based on physician notation in the medical record. The authors concluded that the 5-aminosalicylates would also likely provide this same protection, but would require further investigation.

Eaden et al. found in a separate case-control study that mesalamine at a dose of $\geq 1.2 \mathrm{~g} /$ day reduced colorectal cancer risk by $91 \%$ in patients with UC compared with no treatment (21). There was also a protective effect of more than two visits to the physician per year, but not for colonoscopies.

In conclusion, in a tertiary care center where patients are presumed to be more motivated and better educated regarding their disease, we found a surprisingly low level of medication adherence. From this study, we have shown that men and nonmarried persons are at greatest risk for nonadherent behavior. As this was a prevalence study with the aim to quantify and identify risk factors for medication nonadherence, no data on the consequences of nonadherent behavior was collected. Future prospective studies are warranted to determine the effect of nonadherence on disease course.

\section{ACKNOWLEDGMENTS}

This research was funded by both Procter and Gamble, Cincinnati, OH, and the Reva and David Logan Center for Gastrointestinal Clinical Research.

Reprint requests and correspondence: Sunanda Kane, M.D., M.S.P.H., Assistant Professor of Medicine, University of Chicago, 5841 South Maryland Avenue, MC 4076, Chicago, IL 60637. Received Jan. 4, 2001; accepted May 29, 2001.

\section{REFERENCES}

1. Silverstein M, Lashner B, Hanauer S. Cigarette smoking and ulcerative colitis: A case control study. Mayo Clinic Proc 1994;69:425-9.

2. Fraga X, Vergara M, Medina C, et al. Effects of smoking on the presentation and clinical course of inflammatory bowel disease. Eur J Gastroenterol Hepatol 1997;9:683.

3. Evans JM, McMahon AD, Murray FE, et al. Non-steroidal antiinflammatory drugs are associated with emergency hospital admission to hospital for colitis due to inflammatory bowel disease. Gut 1997;40:619-22.

4. Felder J, Korelitz B, Rajapakse R, et al. Effect of nonsteroidal anti-inflammatory drugs on inflammatory bowel disease: A case-control study. Am J Gastroenterol 2000;95:1949-54.

5. Levenstein S, Prantera C, Varro V. Stress and exacerbation in ulcerative colitis: A prospective study of patients enrolled in remission. Am J Gastroenterol 2000;95:1213-20.

6. The Mesalamine Study Group. An oral preparation of mesalamine as long-term maintenance therapy for ulcerative colitis. A randomized, placebo-controlled trial. Ann Intern Med 1996;124:204-11.

7. Ardizzone S, Petrillo M, Molteni P, et al. Coated oral 5-aminosalicylic acid (Claversal) is equivalent to sulfasalazine for remission maintenance in ulcerative colitis. A double-blind study. J Clin Gastroenterol 1995;21:287-9.

8. Fockens P, Mulder CJ, Tytgat GN, et al. Comparison of the efficacy and safety of 1.5 compared with $3.0 \mathrm{~g}$ oral slowrelease mesalazine (Pentasa) in the maintenance treatment of ulcerative colitis. Dutch Pentasa Study Group. Eur J Gastroenterol Hepatol 1995;7:1025-30.

9. Green JR, Gibson JA, Kerr GD, et al. Maintenance of remission of ulcerative colitis: A comparison between balsalazide $3 \mathrm{~g}$ daily and mesalazine $1.2 \mathrm{~g}$ daily over 12 months. ABA- 
CUS Investigator Group. Aliment Pharmacol Ther 1998;12: 1207-16.

10. Miner P, Hanauer S, Robinson M, et al. Safety and efficacy of controlled-release mesalamine for maintenance of remission in ulcerative colitis. Pentasa UC Maintenance Study Group. Dig Dis Sci 1995;40:296-304.

11. Miller NH. Compliance with treatment regimens in chronic asymptomatic diseases. Am J Med 1997;102:43-9.

12. Truelove SC, Witts LJ. Cortisone in ulcerative colitis: Final report on a therapeutic trial. BMJ 1955;2:1041-4.

13. Irvine EJ, Zhou Q, Thompson AK, and the CCRPT Investigators. The Short Inflammatory Bowel Disease Questionnaire: A quality of life instrument for community physicians managing inflammatory bowel disease. Am J Gastroenterol 1996; 91:1571-8.

14. Steiner J, Koepsell TD, Fihn SD, Inui TS. A general method of compliance assessment using centralized pharmacy records. Med Care 1988;26:814-23.

15. Guyatt GH, Sackett DL, Cook DJ, for the Evidence-Based Medicine Working Group. User's guides to the medical literature. II. How to use an article about therapy or prevention. A.
Are the results of the study valid? JAMA 1993;270:2598601.

16. van Hees PA, van Tongeren JH. Compliance to therapy in patients on a maintenance dose of sulfasalazine. J Clin Gastroenterol 1982;4:333-6.

17. Riley S, Mani V, Goddman MJ, Lucas S. Why do patients with ulcerative colitis relapse? Gut 1990;31:179-83.

18. Weidle $\mathrm{P}$, Ganera $\mathrm{C}$, Irwin $\mathrm{K}$, et al. Adherence to antiretroviral medications in a minority population. J AIDS 1999;22:498502.

19. Willey C, Redding C, Stafford J. Stages of change for adherence with medication regimens for chronic disease: Development and validation of a measure. Clin Ther 2000;22:858-71.

20. Moody GA, Jayanthi V, Probert CS, et al. Long-term therapy with sulphasalazine protects against colorectal cancer in ulcerative colitis: A retrospective study of colorectal cancer risk and compliance with treatment in Leicestershire. Eur J Gastroenterol Hepatol 1996;8:1179-83.

21. Eaden J, Abrams K, Ekbom A, et al. Colorectal cancer prevention in ulcerative colitis: A case-control study. Aliment Pharmacol Ther 2000;14:145-53. 